\title{
Association of Sex, Age, and Comorbidities with Mortality in COVID-19 Patients: A Systematic Review and Meta-Analysis
}

\author{
Mohitosh Biswas $^{a}$ Shawonur Rahaman ${ }^{a}$ Tapash Kumar Biswas ${ }^{b}$ \\ Zahirul Haque $^{c}$ Baharudin Ibrahim ${ }^{d}$ \\ aDepartment of Pharmacy, University of Rajshahi, Rajshahi, Bangladesh; 'bepartment of Medicine, Faridpur \\ Medical College Hospital, Faridpur, Bangladesh; 'Department of Medicine, Rajshahi Medical College Hospital, \\ Rajshahi, Bangladesh; ${ }^{d}$ School of Pharmaceutical Sciences, Universiti Sains Malaysia, Penang, Malaysia
}

\section{Keywords}

Severe acute respiratory syndrome coronavirus-2 .

Coronavirus disease-2019 · Demographic characteristics .

Risk of mortality

\begin{abstract}
Introduction: Although severe acute respiratory syndrome coronavirus- 2 infection is causing mortality in considerable proportion of coronavirus disease-2019 (COVID-19) patients, however, evidence for the association of sex, age, and comorbidities on the risk of mortality is not well-aggregated yet. It was aimed to assess the association of sex, age, and comorbidities with mortality in COVID-2019 patients. Methods: Literatures were searched using different keywords in various databases. Relative risks (RRs) were calculated by RevMan software where statistical significance was set as $p<0.05$. Results: COVID-19 male patients were associated with significantly increased risk of mortality compared to females (RR 1.86: 95\% confidence interval [Cl] 1.67-2.07; $p<$ $0.00001)$. Patients with age $\geq 50$ years were associated with 15.4-folds significantly increased risk of mortality compared to patients with age $<50$ years (RR 15.44: 95\% CI 13.02-18.31; $p<0.00001)$. Comorbidities were also associated with sig-
\end{abstract}

karger@karger.com www.karger.com/int

Karger" nificantly increased risk of mortality; kidney disease (RR 4.90: $95 \% \mathrm{Cl} 3.04-7.88 ; p<0.00001)$, cereborovascular disease (RR $4.78 ; 95 \% \mathrm{Cl} 3.39-6.76 ; p<0.00001)$, cardiovascular disease (RR 3.05: 95\% Cl 2.20-4.25; $p<0.00001$ ), respiratory disease (RR 2.74: 95\% Cl 2.04-3.67; $p<0.00001$ ), diabetes (RR 1.97: $95 \% \mathrm{Cl} 1.48-2.64 ; p<0.00001$ ), hypertension (RR 1.95: 95\% Cl 1.58-2.40; $p<0.00001$ ), and cancer (RR $1.89 ; 95 \% \mathrm{Cl} 1.25-$ $2.84 ; p=0.002$ ) but not liver disease (RR 1.64: $95 \% \mathrm{Cl} 0.82$ 3.28; $p=0.16)$. Conclusion: Implementation of adequate protection and interventions for COVID-19 patients in general and in particular male patients with age $\geq 50$ years having comorbidities may significantly reduce risk of mortality associated with COVID-19.

(c) 2020 S. Karger AG, Basel

\section{Introduction}

Over 6.0 million confirmed cases of severe acute respiratory syndrome coronavirus-2 (SARS-CoV-2)-infected disease called coronavirus disease-2019 (COVID-19) have been detected globally in which over 350,000 deaths occurred at the end of June 2, 2020, as declared by the World Health Organization (WHO). However, it is 
alarming that in the last $24 \mathrm{~h}$ as reported by the WHO on June 2, 2020, 113,198 new confirmed cases of COVID-19 have been detected where 4,242 new deaths had occurred [1]. At present, COVID-19 existence was confirmed in over 200 countries or territories, which indicates that SARS-CoV-2 is rapidly evolving and progresses exponentially throughout the world and is predicted to cause huge morbidity and mortality if the progression is not halted immediately. This may consequently lead to huge socioeconomic global impacts and may also fabricate high burden on the health-care resources.

Since detection of SARS-CoV-2 in early December 2019 in Wuhan, China, the epidemiological, demographic, and clinical features of this pandemic virus have started to appear in the literature. However, it is not well established whether the patient's sex, age, or comorbid diseases were associated with greater risk of SARS-CoV-2 induced severe adverse clinical outcome, for example, mortality. As SARS-CoV-2 is rapidly spreading all over the world, it is predicted that patient's sex, age, and comorbidity might render these patients more vulnerable to either increased mortality or increased risk of infection. Some studies showed that COVID-19 was higher in males than females [2-4], while others did not show similar findings $[5,6]$. Similarly, it is replicated in some studies that patients comparatively older than $\geq 50$ years had high rate for COVID-19-confirmed cases [3,7], while other studies did not get similar findings $[8,9]$. Prevalence of comorbidities in patients with COVID-19 was also highly variable in many studies [10].

Since the global outbreak of SARS-CoV-2 is declared as pandemic by the $\mathrm{WHO}$, appropriate interventions and safety protocols is desperately needed to combat natural infectious challenges. However, patient's sex, age, and comorbidity may act as triggers for increased risk of either infection or mortality caused by COVID-19. Accordingly, a systematic review with meta-analysis considering reasonably larger sample sizes by integrating published studies to date is urgently warranted to establish robust evidence regarding these associations. Therefore, this study was designed to assess the association of sex, age, or comorbidities with risk of mortality by estimating aggregated risk in patients with COVID-19.

\section{Methods}

\section{Literature Search}

This meta-analysis was conducted following Preferred Reporting Items for Systematic Reviews and Meta-Analyses (PRISMA) guidelines as described elsewhere [11]. Literatures were searched in PubMed from February 2, 2020, to May 21, 2020, using keywords as "2019 novel corona virus" or "SARS-CoV-2" or "COVID-19" AND "death or mortality or clinical outcomes or clinical features or epidemiological characteristics.” Additionally, important journal Web sites, for example, New England Journal of Medicine, Journal of American Medical Association, Lancet, Nature, British Medical Journal, etc. were also searched for identifying relevant studies.

\section{Eligibility of Included Studies}

The studies were included based on the following criteria: (i) studies must report data on at least any of these variables, for example, sex, age, or comorbidity with mortality; (ii) sample size of study must be $\geq 102$ due to increased effect on size and statistical power as there were huge number of studies appeared in the literature; (iii) they must be observational studies either retrospective or prospective in nature; (iii) they must be peer-reviewed original published research article; and (iv) articles must be written in English.

\section{Exclusion Criteria}

Studies were excluded if (i) it was a case report, review, viewpoint, perspective, correspondence, letter to the editor, or systematic review; (ii) randomized clinical trials were not considered due to different study design; (iii) study reporting outcomes such as severe/moderate clinical conditions, using mechanical ventilation, admitted to intensive care unit, or critically ill patients was excluded; and (iv) studies reporting clinical data on children or pregnant women were excluded.

\section{Data Extraction, Validity, and Quality Assessment}

Rayyan QCRI, a systematic review software tool [12], was used for primary selection of studies after importing all literature search histories in this software following inclusion and exclusion criteria of current study. For final selection of studies, full texts of all primarily included studies were retrieved and were checked one by one. The selection processes were carried out by 2 investigators MB and SR independently and if any disagreement arose, it was then resolved by the principle investigator via discussion with senior authors. When studies were finally selected, the full text was carefully checked for validity and quality assessment purposes as described below.

For observational cohort studies, Newcastle Ottawa Scale guidelines were followed to determine the quality of included studies, as described elsewhere [13]. All data extraction, input, and analysis were carried out by $\mathrm{MB}$ and was double checked and validated by SR and BI, respectively.

\section{Statistical Analysis}

Relative risks (RRs) and 95\% confidence interval (CI) was calculated using either random or fixed-effect model based on the levels of heterogeneity of the included studies. Heterogeneity in the forest plot was evaluated by using the Cochrane $\chi^{2}$-based Q-test, and regarded as significant if $p$ value $<0.1$ [14]. Meanwhile, the statistic of $\mathrm{I}^{2}$ was used to efficiently test for the heterogeneity, where $I^{2}<25 \%, I^{2}=25-50 \%$, and $I^{2}>50 \%$ indicates low, moderate, and high degree of heterogeneity, respectively [15]. Random-effect model was used to estimate pooled effects if $I^{2}>50$ and fixed-effects model was applied to calculate pooled effects when $I^{2}<50$. Sensitivity analyses were carried out to measure any significant 
Fig. 1. PRISMA flowchart for identifying studies for this systematic review and meta-analysis. PRISMA, Preferred Reporting Items for Systematic Reviews and MetaAnalyses.

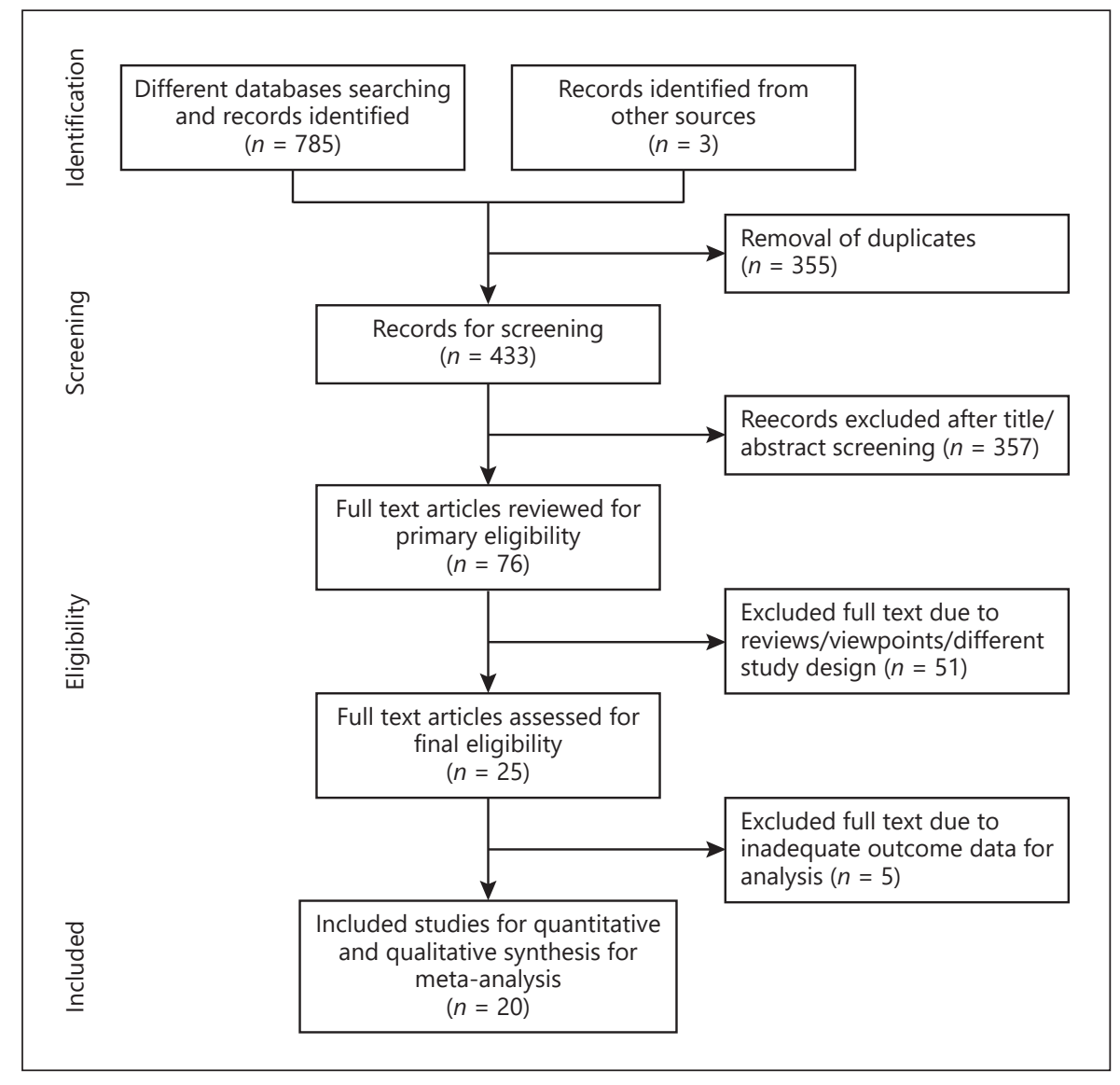

risk differences in-between the studies especially if heterogeneity was found. This was accomplished by removing the included study one after another. In addition, publication bias was carried out by the visual inspection of funnel plots where symmetrical distribution of the plots indicated the absence of publication bias [16]. Funnel plot asymmetry was also assessed with Egger's test [17]. Review-Manager software (RevMan version 5.3 Windows; The Cochrane Collaboration, Oxford, UK) was used for analyzing all data, where the level of statistical significance was set as $<0.05$ (2-sided).

\section{Results}

\section{General Characteristics and Quality of Included Studies}

Flowchart for the identification of included studies in this meta-analysis is shown in Figure 1. In total, 20 studies comprising of 64,676 COVID-19 patients were included in this analysis. It was identified that majority of these studies $(n=16)$ were China-based although one study was identified from Italy, two from the USA, and one study was multinational [18-37]. The important baseline char- acteristics of included studies are summarized in Table 1. The quality of included observational studies either retrospective or prospective in nature as assessed by the Newcastle Ottawa Scale was of high quality (score ranges between 6 and 8) as shown in online suppl. Table 1 (see www.karger.com/doi/10.1159/000512592 for all online suppl. material).

\section{Association of Sex with Mortality in Patients with COVID-19}

As shown in Figure 2, the results of this meta-analysis after pooled risk estimation indicated that male patients with COVID-19 were associated with significantly increased risk of mortality as compared to female patients (RR 1.86: 95\% CI 1.67-2.07; $p<0.00001$ ). Interestingly, when compared sex with SARS-CoV-2 test positivity, it was found that male patients were associated with only $28 \%$ significantly increased risk of confirmed infection by the pandemic SARS-CoV-2 than female patients (RR 1.28: $95 \%$ CI 1.11-1.47; $p=0.0006$; figure not shown here). 


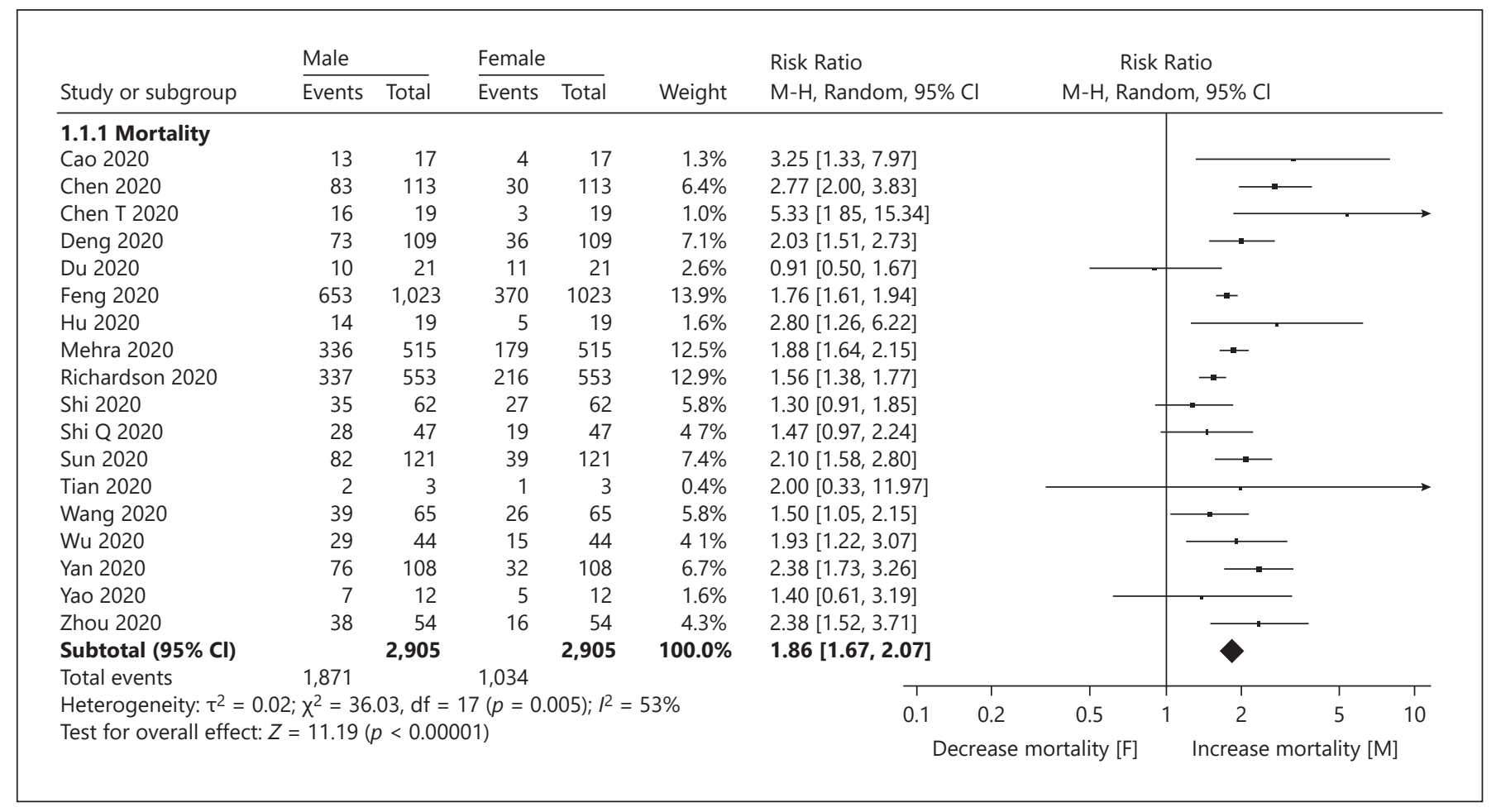

Fig. 2. Forest plot of the pooled effects of COVID-19 male patients against female patients on the risk of mortality. M, male; F, female; CI, confidence interval; COVID-19, coronavirus disease-2019.

Table 1. Baseline characteristics of included studies

\begin{tabular}{|c|c|c|c|c|c|c|}
\hline Cao et a. [18] & China & Retrospective study & 102 & $53(52.0)$ & $54(37-67)$ & 30 \\
\hline Chen et al. [19] & China & Retrospective study & 274 & $171(62.4)$ & $62(44-70)$ & 30 \\
\hline Chen et al. [20] & China & Retrospective study & 203 & $108(53.2)$ & $54(20-91)$ & 41 \\
\hline Feng et al. [24] & China & Observational study & 44,672 & $22,981(51.4)$ & na & 43 \\
\hline Grasselli et al. [36] & Italy & Retrospective study & 1,591 & $1,304(82.0)$ & 63.0 & 34 \\
\hline Hu et al. [23] & China & Retrospective study & 105 & $54(51.4)$ & $60.8 \pm 16.3$ & 29 \\
\hline Mehra et al. [32] & Multinational & Observational study & 8,910 & $5,339(59.9)$ & $52.3 \pm 15.9$ & 40 \\
\hline Palaiodimos et al. [33] & USA & Retrospective study & 200 & $98(49.0)$ & $64(50-73.5)$ & 35 \\
\hline Richardson et al. [37] & USA & Retrospective study & 5,700 & $3,437(60.3)$ & 63.0 & 35 \\
\hline Wang et al. [27] & China & Retrospective study & 339 & $166(49.0)$ & 69.0 & 37 \\
\hline Wu et al. [28] & China & Retrospective study & 201 & $128(63.7)$ & $51(43-60)$ & 51 \\
\hline Yan et al. [29] & China & Retrospective study & 193 & $114(59.1)$ & 64.0 & 45 \\
\hline Yao et al. [30] & China & Retrospective study & 108 & $43(39.8)$ & 52.0 & 12 \\
\hline Zhou et al. [31] & China & Retrospective study & 191 & $119(62.3)$ & $56(46-67)$ & 34 \\
\hline
\end{tabular}

na, not available. 


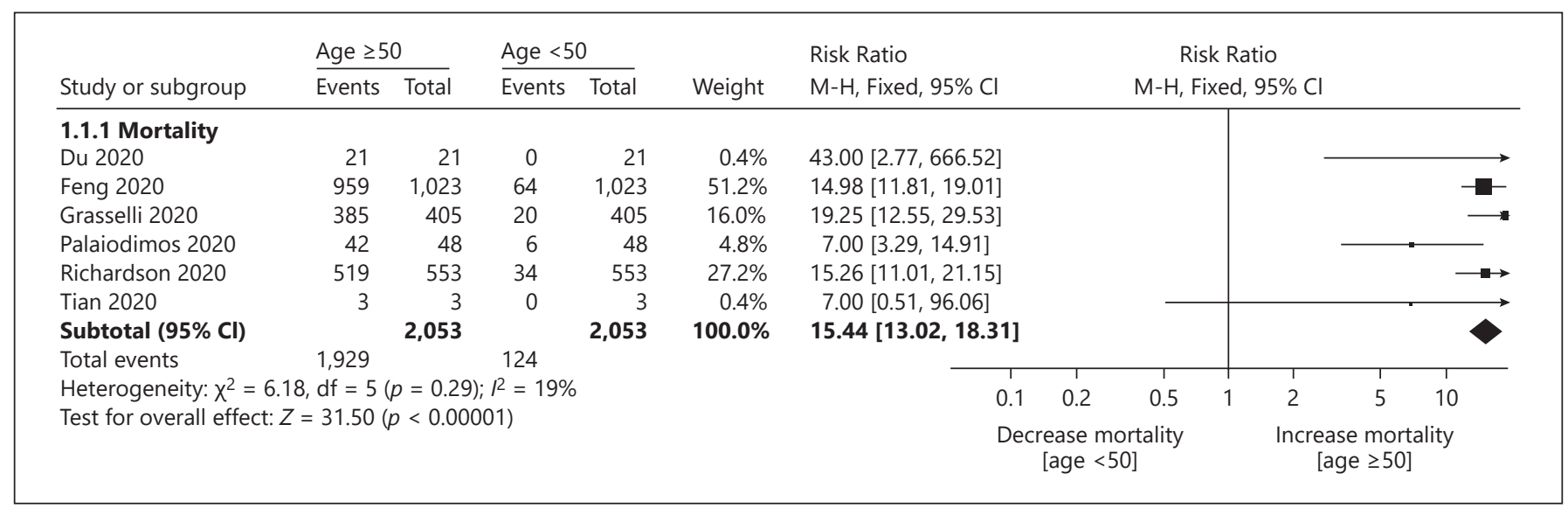

Fig. 3. Forest plot of the pooled effects of COVID- 19 patients with age $\geq 50$ years against patients with age $<50$ years on the risk of mortality. Decre, decrease; Incre, increase; CI, confidence interval; COVID-19, coronavirus disease-2019.

\section{Association of Age with Mortality in Patients with COVID-19}

It was extremely difficult to find age ranges to assess risk of mortality since there was wide variability in reporting clinical outcomes with age ranges. Out of 20 included studies, only 6 studies reported clinical outcomes in such a way that facilitates grouping patients into two arms in which one arm had age $<50$ years, while the other arm had age $\geq 50$ years. No other suitable age ranges were found to consider for analysis.

It was found that patients with age $\geq 50$ years confirmed with SARS-CoV-2 infection were associated with 15.4-folds significantly increased risk of mortality as compared to patients with age $<50$ years (RR 15.44: $95 \%$ CI 13.02-18.31; $p<0.00001$ ), as shown in Figure 3. However, when compared these age-groups with the risk of SARS-CoV-2 infection, it was found that patients with age $\geq 50$ years were associated with only 3.45 -folds significantly increased risk of SARS-CoV-2 test positivity compared to patients with age $<50$ years (RR 3.45: 95\% CI $1.67-7.14 ; p=0.0008$; figure not shown here).

\section{Association of Comorbidities with Mortality in \\ Patients with COVID-2019}

Effects of various comorbidities such as hypertension, diabetes mellitus, respiratory disease, cardiovascular disease, cereborovascular disease, kidney disease, liver disease, and cancer were compared between survivors and non-survivors with these comorbidities. All of these comorbid conditions except liver disease were significantly higher in non-survivors compared to survivors as thus; hypertension (RR 1.95: 95\% CI 1.58-2.40; $p<0.00001$ ), dia- betes (RR 1.97: 95\% CI 1.48-2.64; $p<0.00001$ ), and respiratory disease (RR 2.74: 95\% CI 2.04-3.67; $p<0.00001$; shown in Fig. 4a); cardiovascular disease (RR 3.05: 95\% CI $2.20-4.25 ; p<0.00001)$ and cereborovascular disease (RR 4.78; 95\% CI 3.39-6.76; $p<0.00001$; shown in Fig. 4b); kidney disease (RR 4.90: 95\% CI 3.04-7.88; $p<0.00001$ ), liver disease (RR 1.64: 95\% CI 0.82-3.28; $p=0.16$ ), and cancer (RR 1.89; 95\% CI 1.25-2.84; $p=0.002$; shown in Fig. 4c).

These results indicated that COVID-19 patients with these comorbidities except for liver disease had significantly increased risk of mortality. The mean prevalence of mortality associated with COVID-19 as identified with included studies was $5.1 \%$. Since SARS-CoV-2 is rapidly evolving and has extreme capacity to progress exponentially to the mass community, if this mortality rate is predictively extra-plotted to the general population from all over the world, it may reasonably assume that this may cause huge mortality throughout the world.

\section{Heterogeneity, Sensitivity Analysis, and Publication \\ Bias}

There was significant heterogeneity in studies that tested mortality for sex, hypertension, diabetes, or cardiovascular disease. Moderate heterogeneity was found for studies that tested mortality for age or respiratory disease. However, heterogeneity completely disappeared in studies that tested mortality for cereborovascular disease, kidney disease, liver disease, or cancer. Sensitivity analysis as performed by removing study one after another indicated that no individual study excessively influenced the overall pooled effect in this analysis. From the visual inspection of funnel plot, no publication bias was detected as shown 


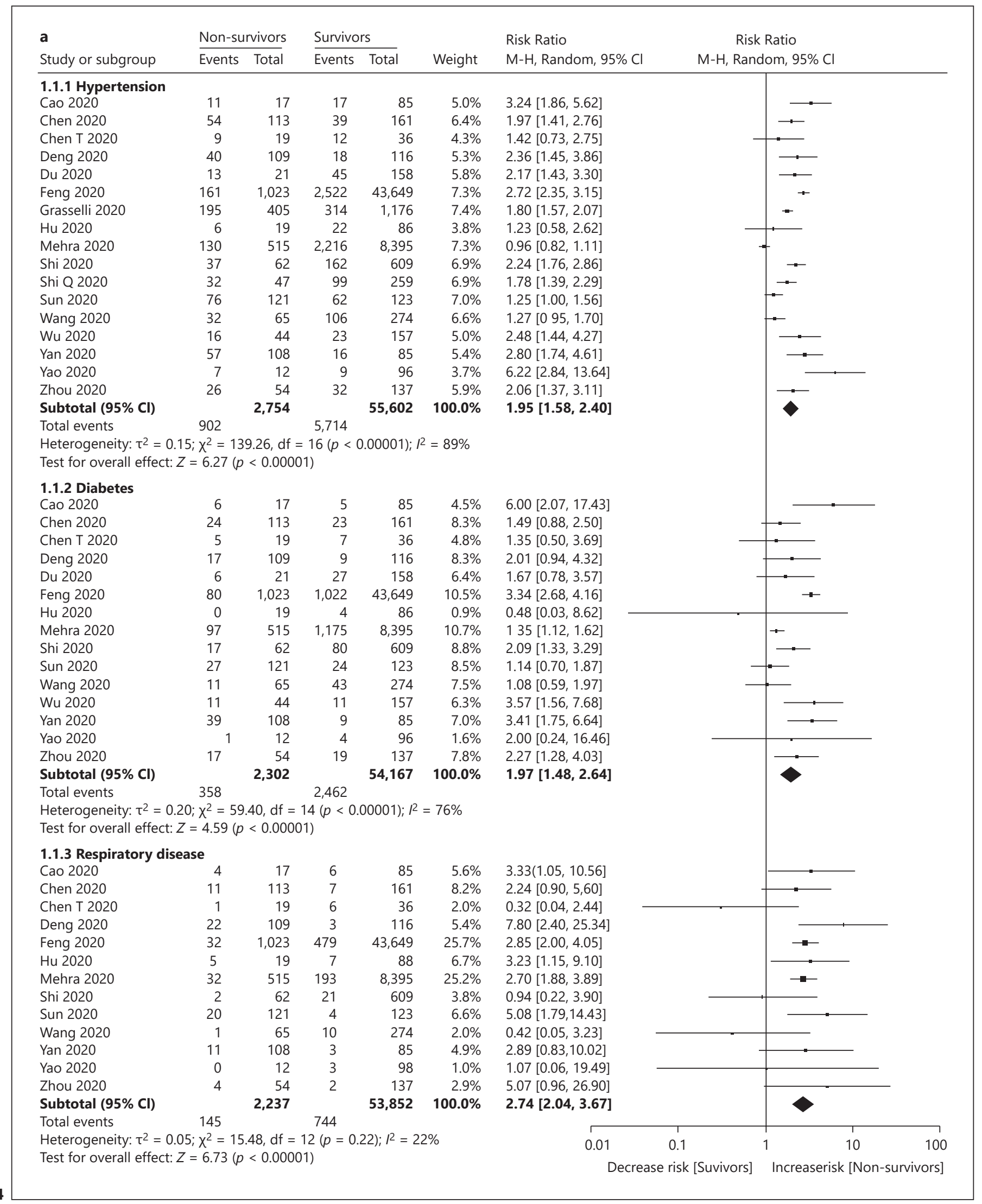

(Figure continued on next page.) 


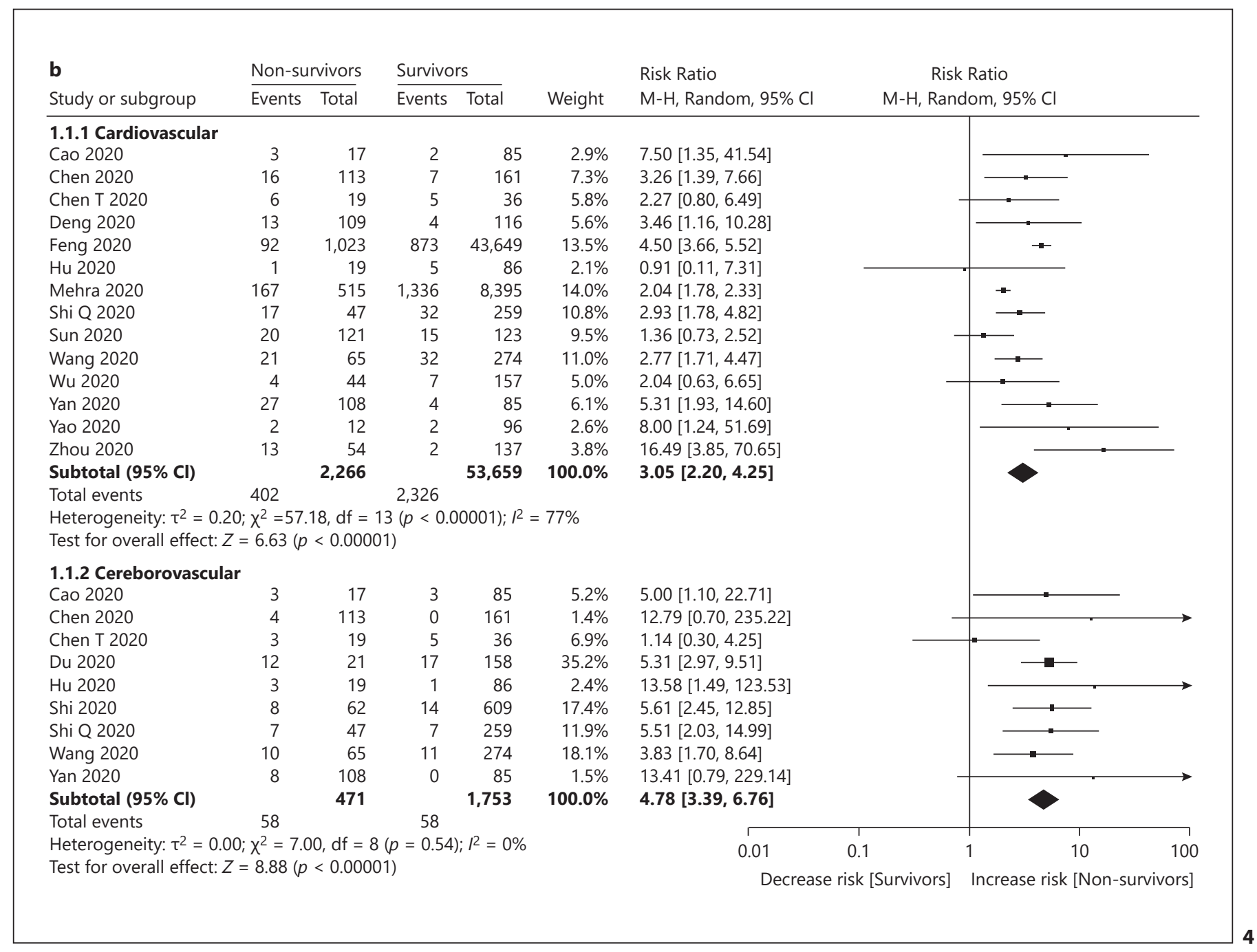

(Figure continued on next page.)

in Figure 5. Analysis revealed that the funnel plot was symmetric and Egger's regression tests provided no evidence of substantial publication bias for either the prevalence of SARS-CoV-2 test positivity among COVID-19 patients $(B=1.7116,95 \% \mathrm{CI}-2.28$ to $5.7, p$ value $=0.3767)$ or for hypertension with mortality in patients with $\mathrm{CO}$ VID-19 $(B=1.3765,95 \%$ CI -1.89 to 4.6 , $p$ value $=0.3817)$. The results showed that Egger's test from two different variables (SARS-CoV-2 test positivity or hypertension) were consistent with $p>0.05$, indicating no significant publication bias in this study. In summary, male patients, age $\geq 50$ years, or had comorbid conditions (e.g., kidney disease, cereborovascular disease, cardiovascular disease, respiratory disease, diabetes, hypertension, and cancer) were significantly associated with increased risk of mortality in COVID-19.

\section{Discussion}

Pandemic COVID-19 is causing huge morbidity and mortality throughout the world; however, the association of sex, age, or comorbidities with mortality has not been investigated in larger effect size. To the very best of the authors' knowledge to date, this is the 1st study to estimate aggregated risk of gender, age, or comorbidity with mortality in patients with COVID-19. The findings may be considered as novel providing evidence that male patients, age $\geq 50$ years, or had comorbidities were significantly associated with increased risk of mortality. Since risk of mortality was assessed for considerably large number of high-quality data of COVID-19 patients with different interventions, the evidence may, therefore, be considered as high standard. 


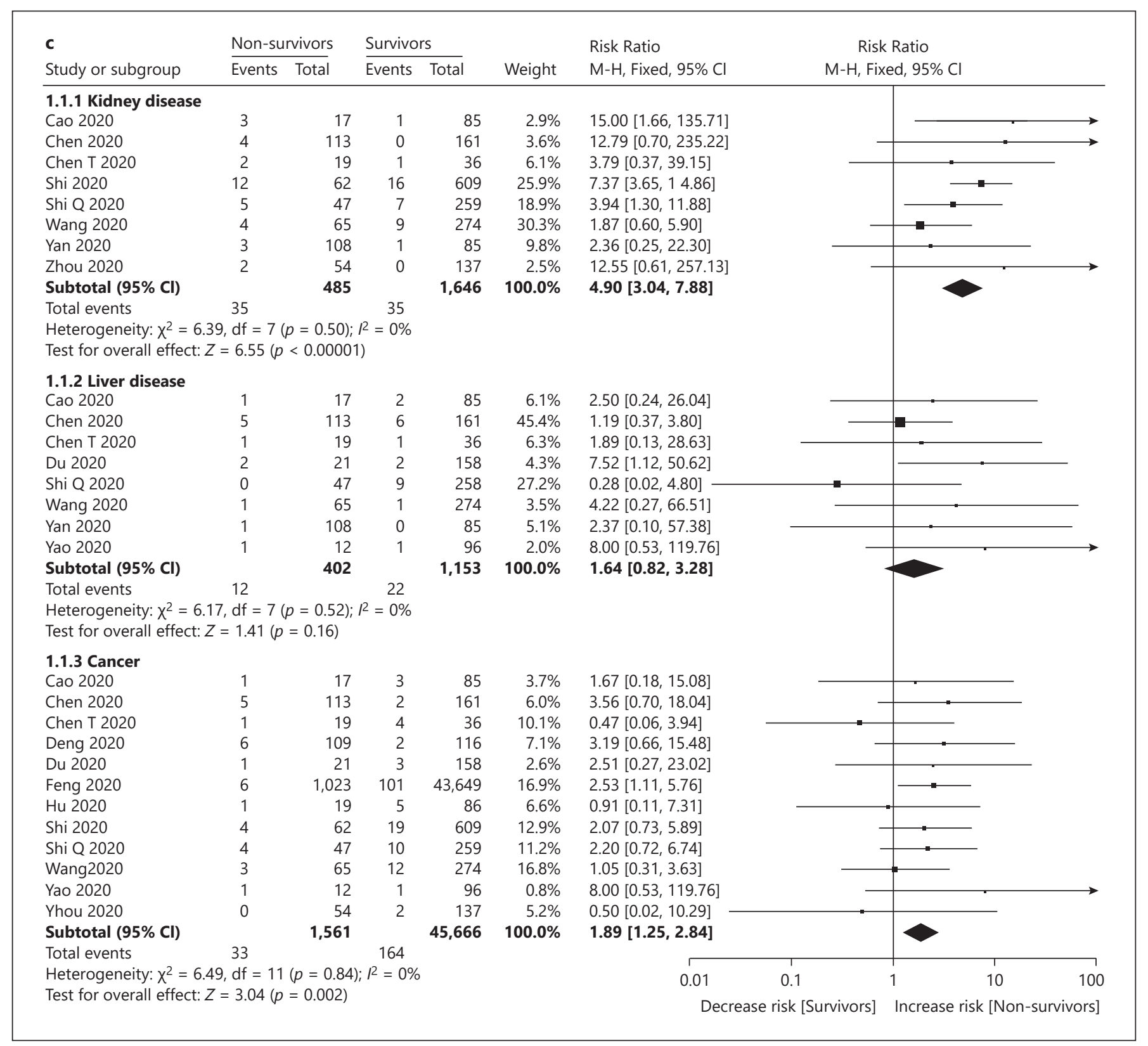

Fig. 4. a Forest plot of the pooled effects of comorbidities on the risk of mortality associated with hypertension, diabetes or respiratory disease. $\mathbf{b}$ Forest plot of the pooled effects of comorbidities on the risk of mortality associated with cardiovascular or cereborovascular disease. c Forest plot of the pooled effects of comorbidities on the risk of mortality associated with kidney disease, liver disease, or cancer. Incre, increase; CI, confidence interval.

Without elucidation exact mechanism and confirmation, it may not plausible to explain why mortality was significantly higher in male COVID-19 patients than females. As reviewed elsewhere, it has been stated that male patients may had higher expression of angiotensin-converting enzyme 2 (ACE2), which may be regulated by male sex hormones rendering them to more risk for SARS-CoV-2 infection and poor clinical outcomes as well [38]. In addition, this may be partly because ACE2 expression encoded by the ACE2 gene lays on the X-chromosome, thus allowing females to be potentially heterozygous whereas men who are definitely homozygous al- 


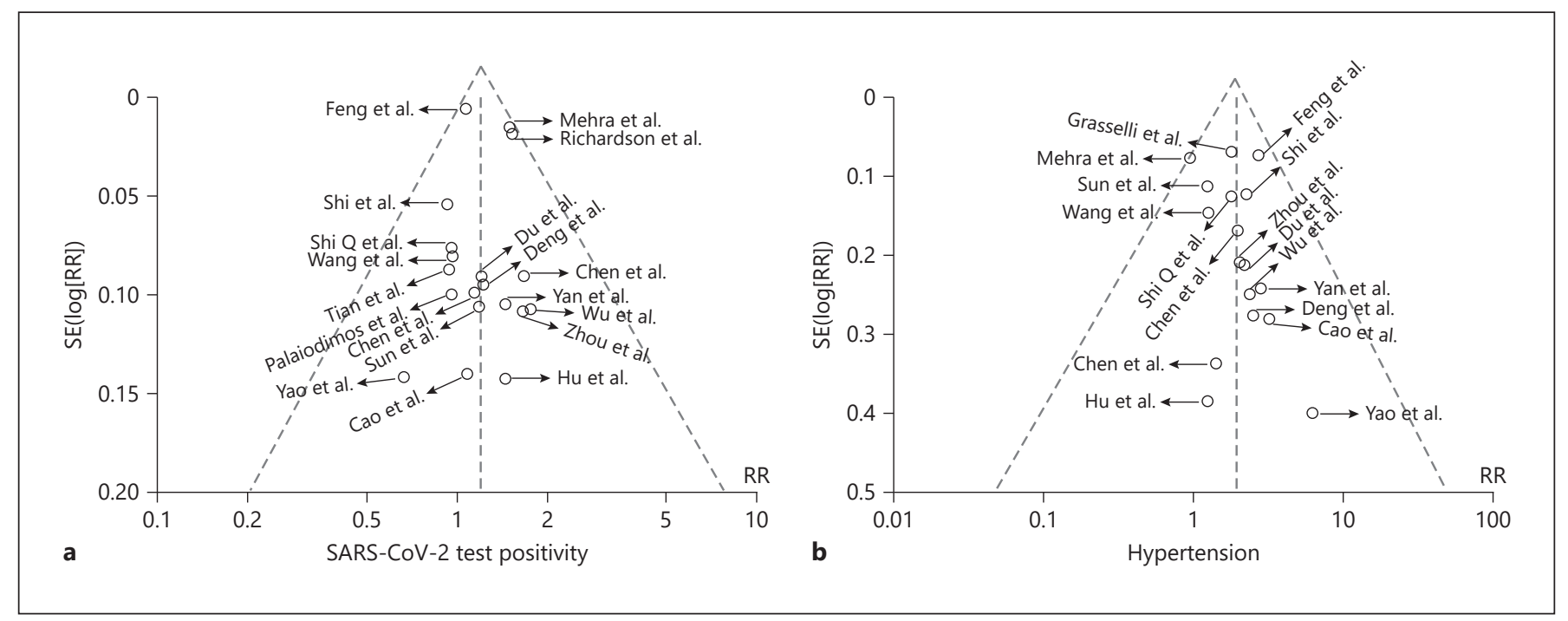

Fig. 5. Funnel plot for detection of publication bias as determined by Egger's test. a Funnel plot for COVID-19 patients positive for SARS-CoV-2 test. b Funnel plot for COVID-19-hypertensive patients and risk for mortality. COVID-19, coronavirus disease-2019; SARS-CoV-2, severe acute respiratory syndrome coronavirus-2.

lowing males to be potentially high ACE2 expressor [39]. Moreover, it is hypothesized that females may counteract the progression of the SARS-CoV-2 infection and severe clinical outcomes due to carrying $\mathrm{X}$-linked heterozygous alleles called sex dimorphism by activating a mosaic advantage [39]. Contrastingly, some authors stated that ACE2 expression may act as a biological sword and may protect patients from organ injury. It has also been postulated that the ACE2 upregulation may improve clinical outcomes in SARS-CoV-2-infected patients by protecting organ injury, although not specifying any gender-oriented effects [40, 41]. Amid the conflicting evidence regarding the association of ACE2 expression with the magnitude of SARS-CoV-2 infection and clinical outcomes, the findings of present analysis warrants future clinical studies assessing gender specific effects of ACE2 expression in relation to mortality or any other underlying reasons causing poor clinical outcome in both sexes with COVID-19, which may be influenced by age also.

There is correlation between age and natural immunity as reviewed elsewhere and concluded that older people are particularly prone to develop more infections as natural immunity declines gradually at older ages [42]. Older people are also vulnerable to adverse drug reactions which may be partly because of the either reduced organ function at older age or taking multiple drugs due to comorbidities [43]. The results of the current study indicated that people aged 50 years or older were significant- ly at higher risk of mortality than those younger than 50 years. Linear regression with ACE2 gene expression in nasal epithelium as the dependent variable and age-group as the independent variable, a recent study showed that compared with younger children, $A C E 2$ gene expression was significantly higher in older children $(p=0.01)$, young adults $(p<0.001)$, and adults $(p=0.001)$ which may partly able to explain why mortality was significantly higher in older patients, as identified in present study [44]. Based on this evidence and from the findings of the current study, it is postulated that patients older than 50 years may have higher expression of ACE2 encoded by the ACE2 gene as well as have other conventional factors, for example, reduced immunity, low organ function, or coexisting comorbidities which may be accountable for greatly increased risk of mortality.

Comorbidity may also relate to reduced immune function. For example, in diabetic patients, natural immune function reduced substantially which may restrict the body to produce respective antibody against any infection [45]. Also, polypharmacy and comorbidity are interrelated and dependable to each other. Since natural immunity is declined profoundly in comorbid conditions and as patients are taking more drugs concurrently, the notorious adverse drug reactions (ADRs) alongside downregulation of immune function may expected to occur in these patients and may increase risk of mortality eventually. 
It is also known that after the transmission of SARS$\mathrm{CoV}-2$ into the human body, ACE-2 receptor accelerates the binding of this pathogenic virus to their target cells. The ACE-2 is highly expressed in epithelial cells of the lung, intestine, kidney, and blood vessels which is predominantly upregulated in patients with either diabetes or hypertension treating with ACE inhibitors (ACEIs) and angiotensin II type-I receptor blockers (ARBs) [46, 47]. This upregulation of ACE- 2 in patients with diabetes and hypertension treating ACEIs or ARBs may therefore exacerbate infection with SARS-CoV-2. It is therefore hypothesized by some authors [46-48] that diabetes and hypertensive patients may eventually increase risk of developing severe and fatal COVID-19, which is in line with findings of the current study.

The results of this analysis found that comorbidities were significantly aggravating mortality in SARS-CoV-2 infection. In contrast, a recent meta-analysis found that using ACEIs/ARBs in COVID-19 patients were not associated with higher risk of mortality or severity of infection [49]. Since both hypertension and diabetes independently associated with significantly increased risk of mortality, the findings of current study suggest extremely close monitoring for COVID-2019 patients treated with ACEIs/ARBs, even if possible, may be treated with alternative cardiovascular medicines. For example, Fang et al. [46] suggest to use calcium channel blockers instead of ACEIs/ARBs as did not find any evidence for increased ACE-2 expression or activity by calcium channel blockers. However, in these clinical debating situations, professional societies recommended to continue ACEIs/ARBs in COVID-19 patients unless it is contraindicated for other clinical conditions rather than SARS-CoV-2 infection [50].

Each comorbidity has its own pathophysiology and mechanistic link between SARS-CoV-2 infection and mortality, some of which has already been proposed or established while others unrevealed yet. For example, chronic kidney disease (CKD) has been associated with inflammation and dysregulation of immune function which may explain increased risk of mortality in COVID-19 patients with kidney disease [51]. It has been reported in a recent study that ACE2 receptor is overexpressed in the tubular cells of COVID-19 patients with kidney disease characterized by increased serum creatinine and urea nitrogen $[51,52]$. Taken together, the alterations in ACE2 receptor expression and dysregulation of immune function may answer to why the highest significantly increased risk of mortality was found in COVID-19 patients with kidney disease in the present study.
Also, increased ACE2 expression at both mRNA and protein levels was found in patients with basic heart failure disease, indicating that if infected by SARS-CoV-2, these patients may have greater risk of heart attack and consecutively adverse clinical outcomes [53]. This is also in line with the current study findings that show COVID-19 patients with cardiovascular disease were associated with significantly increased risk of mortality.

Although present analysis found a strong association of age $\geq 50$ years with mortality and also comorbidities with mortality as well, it failed to establish the association between age and comorbidities combined with mortality due to lacking reported data regarding these associations. However, it is generally assumed that if COVID-19 patients had age $\geq 50$ years and also had comorbidities, then the risk of mortality would be much greater than exposing either the alone risk factor (age or comorbidity), warranting future clinical studies to explore such associations.

It is found that mean prevalence of mortality was significantly lower than survivors associated with $\mathrm{CO}$ VID-19, however, when predictively extra-plotting this mortality rate to general population, it is predicted that huge number of patients may die due to SARS-CoV-2 infection. Hence, more rigid and strict measures should be immediately implemented in the society in this regard to tackle this natural infectious disaster which may facilitate to save human beings profoundly.

\section{Limitations}

In spite of providing robust evidence for the association of sex, age, or comorbidities with mortality in COVID-19 patients, there are some limitations of this study. First, variables, for example, sex, age, or comorbidities were not adjusted; therefore, the results presented in this study may vary if results were adjusted with these along with other variables affecting clinical outcomes. Second, in some tested variables, there was high level of heterogeneity which may be partly because of different study designs of included studies, patient's characteristics, various treatments, ethnicity, or unadjusted variables. Third, as compared to real death toll from all over the world caused by COVID-19, data presented in this study are relatively small due to not getting outcome data yet. Having those limitations, it is expected that the robust evidence provided in this study may play a pivotal role to stakeholders, policymakers, and health-care professionals to intervene the greatest infectious and unprecedented health challenges of 21st century and may advance epidemiologic features, disease progression, and clinical outcomes associated with SARS-CoV-2 infection. 


\section{Conclusions}

Male patients with COVID-19 were associated with significantly increased risk of mortality compared to females. Patients with age $\geq 50$ years were at a significantly massive risk of mortality compared to those aged $<50$ years. Mortality was significantly higher in those patients with kidney disease, cereborovascular disease, cardiovascular disease, respiratory disease, diabetes, hypertension, and cancer. Implementation of adequate protection and interventions for COVID-19 patients in general and in particular male patients with age $\geq 50$ years having comorbidities may significantly reduce the risk of mortality associated with COVID-19.

\section{Acknowledgement}

The principal investigator, M.B., greatly acknowledges Professor Liz Milward at the School of Biomedical Sciences and Pharmacy in the University of Newcastle, Australia.

\section{Statement of Ethics}

The paper is exempt from Ethical Committee approval since it is a systematic review and meta-analysis.

\section{Conflict of Interest Statement}

The authors declare no conflict of interest.

\section{Funding Sources}

No funding was available for this research.

\section{Author Contributions}

M.B. conceived and designed this study and performed all statistical analysis. M.B. and S.R. were involved in the selection process of studies. Data extraction and analysis were double checked by S.R. and B.I., respectively. M.B. drafted this article and B.I., M.Z.H., and T.K.B. reviewed critically for the improvement of the overall quality of this manuscript, and all authors agreed to submit the manuscript.

\section{References}

1 WHO. Coronavirus disease (COVID-2019) situation reports [Internet]. 2020 [cited 2020 Jun 2].

2 Huang C, Wang Y, Li X, Ren L, Zhao J, Hu Y, et al. Clinical features of patients infected with 2019 novel coronavirus in Wuhan, China. Lancet. 2020;395(10223):497-506.

3 Yang X, Yu Y, Xu J, Shu H, Xia J, Liu H, et al. Clinical course and outcomes of critically ill patients with SARS-CoV-2 pneumonia in Wuhan, China: a single-centered, retrospective, observational study. Lancet Respir Med. 2020 May;8(5):475-81.

4 Chen N, Zhou M, Dong X, Qu J, Gong F, Han $\mathrm{Y}$, et al. Epidemiological and clinical characteristics of 99 cases of 2019 novel coronavirus pneumonia in Wuhan, China: a descriptive study. Lancet. 2020;395(10223):507-13.

$5 \mathrm{Xu} \mathrm{X,} \mathrm{Yu} \mathrm{C,} \mathrm{Qu} \mathrm{J,} \mathrm{Zhang} \mathrm{L,} \mathrm{Jiang} \mathrm{S,} \mathrm{Huang} \mathrm{D,}$ et al. Imaging and clinical features of patients with 2019 novel coronavirus SARS-CoV-2. Eur J Nucl Med Mol Imaging. 2020 May; 47(5):1275-80.

6 Qian G-Q, Yang N-B, Ding F, Ma AHY, Wang Z-Y, Shen Y-F, et al. Epidemiologic and clinical characteristics of 91 hospitalized patients with COVID-19 in Zhejiang, China: a retrospective, multi-centre case series. QJM. 2020 Jul 1;113(7):474-81.

7 Zhang J, Dong X, Cao Y, Yuan Y, Yang Y, Yan $\mathrm{Y}$, et al. Clinical characteristics of 140 patients infected with SARS-CoV-2 in Wuhan, China. Allergy. 2020 Jul;75(7):1730-41.
$8 \mathrm{Xu} \mathrm{YH}$, Dong JH, An WM, Lv XY, Yin XP, Zhang JZ, et al. Clinical and computed tomographic imaging features of novel coronavirus pneumonia caused by SARS-CoV-2. J Infect. 2020 Apr;80(4):394-400.

9 Wang Z, Yang B, Li Q, Wen L, Zhang R. OUP accepted manuscript. Clin Infect Dis. 2020. DOI: $10.1093 / \mathrm{cid} / \mathrm{ciaa} 272$.

10 Li B, Yang J, Zhao F, Zhi L, Wang X, Liu L, et al. Prevalence and impact of cardiovascular metabolic diseases on COVID-19 in China. Clin Res Cardiol. 2020 May;109(5):531-8.

11 Higgins JPT, Thomas J, Chandler J, Cumpston M, Li T, Page MJ WV. Cochrane Handbook for Systematic Reviews of Interventions version 6.0 (updated July 2019). Cochrane, 2019. [Internet]. Handbook; 2019.

12 Ouzzani M, Hammady H, Fedorowicz Z, Elmagarmid A. Rayyan: a web and mobile app for systematic reviews. Syst Rev. 2016;5(1):210.

13 Wells G, Shea B, O'Connell D, Peterson J. The Newcastle-Ottawa Scale (NOS) for assessing the quality of nonrandomised studies in meta-analyses [Internet]. Ottawa Hosp Res Inst. 2000

14 Lau J, Ioannidis JP, Schmid CH. Quantitative synthesis in systematic reviews. Ann Intern Med. 1997;127(9):820-6.

15 Higgins JP, Thompson SG, Deeks JJ, Altman DG. Measuring inconsistency in meta-analyses. BMJ. 2003;327(7414):557-60.
16 Sutton AJ, Duval SJ, Tweedie RL, Abrams KR, Jones DR. Empirical assessment of effect of publication bias on meta-analyses. BMJ. 2000; 320(7249):1574-7.

17 Egger M, Davey Smith G, Schneider M, Minder C. Bias in meta-analysis detected by a simple, graphical test. BMJ. 1997;315(7109):62934.

18 Cao J, Tu WJ, Cheng W, Yu L, Liu YK, Hu X, et al. Clinical features and short-term outcomes of 102 patients with corona virus disease 2019 in Wuhan, China. Clin Infect Dis. 2020;71(15):748-55.

19 Chen T, Wu D, Chen H, Yan W, Yang D, Chen G, et al. Clinical characteristics of 113 deceased patients with coronavirus disease 2019: retrospective study. BMJ. 2020 Mar 26; 368:m1091.

20 Chen TL, Dai Z, Mo P, Li X, Ma Z, Song S, et al. Clinical characteristics and outcomes of older patients with coronavirus disease 2019 (COVID-19) in Wuhan, China (2019): a single-centered, retrospective study. J Gerontol A Biol Sci Med Sci. 2020 Sep 16;75(9):178895.

21 Deng Y, Liu W, Liu K, Fang YY, Shang J, Zhou $\mathrm{L}$, et al. Clinical characteristics of fatal and recovered cases of coronavirus disease 2019 (COVID-19) in Wuhan, China: a retrospective study. Chin Med J. 2020 Jun 5;133(11): 1261-67. 
22 Du RH, Liang LR, Yang CQ, Wang W, Cao TZ, Li M, et al. Predictors of mortality for patients with COVID-19 pneumonia caused by SARSCoV-2: a prospective cohort study. Eur Respir J. 2020;55(5).

$23 \mathrm{Hu} \mathrm{H}$, Yao N, Qiu Y. Comparing rapid scoring systems in mortality prediction of critical ill patients with novel coronavirus disease. Acad Emerg Med. 2020 Jun;27(6):461-8.

24 Zijian Feng QL, Zhang Y, Wu Z, Xiaoping Dong H, Ma, Yin D, et al. The epidemiological characteristics of an outbreak of 2019 novel coronavirus diseases (COVID-19): China, 2020. CCDC Wkly. 2020;2(8):113-22.

25 Shi S, Qin M, Shen B, Cai Y, Liu T, Yang F, et al. Association of cardiac injury with mortality in hospitalized patients with COVID-19 in Wuhan, China. JAMA Cardiol. 2020 Jul 1; 5(7):802-10.

26 Sun H, Ning R, Tao Y, Yu C, Deng X, Zhao C, et al. Risk factors for mortality in 244 older adults with COVID-19 in Wuhan, China: a retrospective study. J Am Geriatr Soc. 2020 Jun;68(6):E19-23.

27 Wang L, He W, Yu X, Hu D, Bao M, Liu H, et al. Coronavirus disease 2019 in elderly patients: characteristics and prognostic factors based on 4-week follow-up. J Infect. 2020; 80(6):639-45.

28 Wu C, Chen X, Cai Y, Xia J, Zhou X, Xu S, et al. Risk factors associated with acute respiratory distress syndrome and death in patients with coronavirus disease 2019 Pneumonia in Wuhan, China. JAMA Intern Med. 2020 Jul 1; 180(7):934-43.

29 Yan Y, Yang Y, Wang F, Ren H, Zhang S, Shi $\mathrm{X}$, et al. Clinical characteristics and outcomes of patients with severe covid-19 with diabetes. BMJ Open Diabetes Res Care. 2020;8(1).

30 Yao Q, Wang P, Wang X, Qie G, Meng M, Tong X, et al. Retrospective study of risk factors for severe SARS-Cov-2 infections in hospitalized adult patients. Polish Arch Intern Med. 2020 May 29;130(5):390-9.

31 Zhou F, Yu T, Du R, Fan G, Liu Y, Liu Z, et al Clinical course and risk factors for mortality of adult inpatients with COVID-19 in $\mathrm{Wu}$ han, China: a retrospective cohort study. Lancet. 2020 Mar 28;395(10229):1054-62.

32 Mehra MR, Desai SS, Kuy S, Henry TD, Patel AN. Cardiovascular disease, drug therapy, and mortality in Covid-19. N Engl J Med. 2020 Jun 18;382(25):e102.
33 Palaiodimos L, Kokkinidis DG, Li W, Karamanis D, Ognibene J, Arora S, et al. Severe obesity is associated with higher in-hospital mortality in a cohort of patients with COVID-19 in the Bronx. New York: Metabolism; 2020. p. 154262.

34 Shi Q, Zhang X, Jiang F, Zhang X, Hu N, Bimu $\mathrm{C}$, et al. Clinical characteristics and risk factors for mortality of COVID-19 patients with diabetes in Wuhan, China: a two-center, retrospective study. Diabetes Care. 2020;43(7): 1382.

35 Tian S, Hu N, Lou J, Chen K, Kang X, Xiang $\mathrm{Z}$, et al. Characteristics of COVID-19 infection in Beijing. J Infect. 2020 Apr;80(4):4016

36 Grasselli G, Zangrillo A, Zanella A, Antonelli M, Cabrini L, Castelli A, et al. Baseline characteristics and outcomes of 1591 patients infected with SARS-CoV-2 admitted to ICUs of the lombardy region. JAMA. 2020 Apr 28; 323(16):1574-81.

37 Richardson S, Hirsch JS, Narasimhan M Crawford JM, McGinn T, Davidson KW, et al. Presenting characteristics, comorbidities, and outcomes among 5700 patients hospitalized with COVID-19 in the New York City area. JAMA. 2020 May 26;323(20):2052-59.

38 La Vignera S, Cannarella R, Condorelli RA, Torre F, Aversa A, Calogero AE. Sex-specific SARS-CoV2 mortality: among hormonemodulated ace 2 expression, risk of venous thromboembolism and hypovitaminosis $\mathrm{D}$. Int J Mol Sci. 2020;21(8).

39 Gemmati D, Bramanti B, Serino ML, Secchiero P, Zauli G, Tisato V. COVID-19 and individual genetic susceptibility/receptivity: role of ACE1/ACE2 genes, immunity, inflammation and coagulation. Might the double Xchromosome in females be protective against SARS-CoV-2 compared to the single X-chromosome in males? Int J Mol Sci. 2020;21(10): 3474 .

40 Vaduganathan M, Vardeny O, Michel T, McMurray JJV, Pfeffer MA, Solomon SD. Reninangiotensin-aldosterone system inhibitors in patients with Covid-19. N Engl J Med. 2020; 382(17):1653-9.

41 Gurwitz D. Angiotensin receptor blockers as tentative SARS-CoV-2 therapeutics. Drug Dev Res. 2020 Aug;81(5):537-40.

42 Leng J, Goldstein DR. Impact of aging on viral infections. Microbes Infect. 2010;12(14-15): $1120-4$.
43 Lavan AH, Gallagher P. Predicting risk of adverse drug reactions in older adults. Ther Adv Drug Saf. 2016;7(1):11-22.

44 Bunyavanich S, Do A, Vicencio A. Nasal gene expression of angiotensin-converting enzyme 2 in children and adults. JAMA. 2020 Jun 16; 323(23):2427-9.

45 Berbudi A, Rahmadika N, Cahyadi AI, Ruslami R. Type 2 diabetes and its impact on the immune system. Curr Diabetes Rev. 2020; 16(5):442-9.

46 Fang L, Karakiulakis G, Roth M. Are patients with hypertension and diabetes mellitus at increased risk for COVID-19 infection? Lancet Respir Med. 2020 Apr;8(4):e21.

$47 \mathrm{Li} \mathrm{XC}$, Zhang J, Zhuo JL. The vasoprotective axes of the renin-angiotensin system: physiological relevance and therapeutic implications in cardiovascular, hypertensive and kidney diseases. Pharmacol Res. 2017;125(Pt A): 21-38.

48 Wan Y, Shang J, Graham R, Baric RS, Li F. Receptor recognition by novel coronavirus from Wuhan: an analysis based on decadelong structural studies of SARS. J Virol. 2020 Mar 17;94(7):e00127-20.

49 Zhang X, Yu J, Pan LY, Jiang HY. ACEI/ARB use and risk of infection or severity or mortality of COVID-19: a systematic review and meta-analysis. Pharmacol Res. 2020;158:104927.

50 Mehta N, Kalra A, Nowacki AS, Anjewierden S, Han Z, Bhat P, et al. Association of use of angiotensin-converting enzyme inhibitors and angiotensin II receptor blockers with testing positive for coronavirus disease 2019 (COVID-19). JAMA Cardiol. 2020 Sep 1;5(9): 1020-6.

51 Oyelade T, Alqahtani J, Canciani G. Prognosis of COVID-19 in patients with liver and kidney diseases: an early systematic review and meta-analysis. Trop Med Infect Dis. 2020;5(2):80.

52 Zou X, Chen K, Zou J, Han P, Hao J, Han Z. Single-cell RNA-seq data analysis on the receptor ACE2 expression reveals the potential risk of different human organs vulnerable to 2019-nCoV infection. Front Med. 2020;14(2): $185-92$.

53 Chen L, Li X, Chen M, Feng Y, Xiong C. The ACE2 expression in human heart indicates new potential mechanism of heart injury among patients infected with SARS-CoV-2. Cardiovasc Res. 2020;116(6):1097-100. 